\title{
Effect of first-line endocrine therapy in patients with hormone-sensitive advanced breast cancer: a network meta-analysis
}

This article was published in the following Dove Press journal:

OncoTargets and Therapy

Tingting Zhang'
Fubin Feng ${ }^{2}$
Wenge Zhao
Jinhui Tian ${ }^{4}$
Yan Yao ${ }^{3}$
Chao Zhou ${ }^{2}$
Shengjie Dong
Congcong Wang
Chuanxin Zang'
Qingliang Lv
Changgang Sun
',8
'College of Traditional Chinese
Medicine, Shandong University
of Traditional Chinese Medicine,
WeiFang, China; ${ }^{2}$ Department of
Oncology, Weifang Traditional Chinese
Hospital, WeiFang, China; ${ }^{3}$ Department
of Oncology, Clinical Medical
Colleges, Weifang Medical University,
WeiFang, China; ${ }^{4}$ Evidence-Based
Medicine Center, School of Basic
Medical Sciences, Lanzhou University,
Lanzhou, China; ${ }^{5}$ Department of the
Joint and Bone Surgery, Yantaishan
Hospital, Yantai, China; ${ }^{6}$ Department
of Oncology, Zibo Maternal and
Child Health Hospital, Zibo, China;
${ }^{7}$ Department of Radiology, Weifang
people's Hospital, WeiFang, China;
${ }^{8}$ Department of Oncology, Affiliated
Hospital of Weifang Medical
University, WeiFang, China

Correspondence: Changgang Sun Department of Oncology, Affilited Hospital of Weifang Medical University, No. 2428, Yuhe Road, Kuiwen District, WeiFang, ShanDong Province, China Tel +86 I3583690699

Email scgdoctor@।26.com
Background: Endocrine therapy is the cornerstone treatment for patients with hormone receptor-positive advanced breast cancer. We aimed to assess the effectiveness of various first-line endocrine monotherapies or combinations to determine the optimal sequence in a network meta-analysis.

Materials and methods: We searched PubMed, EMBASE, and the Cochrane Library for randomized controlled trials (RCTs) from inception up to November 21, 2017. We included only RCTs that assessed the effectiveness of the following treatments as a monotherapy or in combination as the first-line treatment: tamoxifen, anastrozole, letrozole, exemestane, fulvestrant, palbociclib, and ribociclib. The results were presented with pooled odds ratio or hazard ratio (HR), and $95 \%$ credible interval (CrI). The primary outcomes were objective response rate (ORR) and progression-free survival/time to progression.

Results: A total of 16 eligible articles (14 RCTs) involving 6,602 patients treated with 10 different first-line endocrine therapies were assessed in our network meta-analysis. Palbociclib plus letrozole was superior to anastrozole, letrozole, exemestane, fulvestrant $500 \mathrm{mg}$, and anastrozole plus fulvestrant (loading dose) $(\mathrm{HR}=0.44,95 \% \mathrm{CrI}$ : $0.33-0.58$; $\mathrm{HR}=0.56,95 \% \mathrm{CrI}: 0.45-0.68$; $\mathrm{HR}=0.45,95 \%$ CrI: $0.32-0.61 ; \mathrm{HR}=0.58,95 \%$ CrI: $0.42-0.81 ; \mathrm{HR}=0.50,95 \%$ CrI: $0.37-0.68$; respectively). However, there is no significant advantage compared with ribociclib plus letrozole $(\mathrm{HR}=1.00,95 \% \mathrm{CrI}: 0.72-1.39)$. In terms of ORR, ribociclib plus letrozole is more effective than palbociclib plus letrozole (odds ratio=1.30, 95\% CrI: 0.83-2.02).

Conclusion: Palbociclib plus letrozole and ribociclib plus letrozole might be the optimal firstline endocrine therapeutic choices for hormone receptor-positive/human epidermal growth factor receptor 2-negative advanced breast cancer due to a longer progression-free survival/time to progression and a more efficacious ORR.

Keywords: first-line endocrine therapy, progression-free survival, objective response rate, advanced breast cancer, network meta-analysis, randomized controlled trial

\section{Introduction}

Worldwide, breast cancer is by far the highest incidence of cancer in women and the leading cause of cancer deaths, accounting for $25 \%$ of all cancer cases ( 1.68 million) and $15 \%$ of cancer deaths $(520,000){ }^{1,2}$ Approximately $30 \%-40 \%$ of patients diagnosed at early stages could develop advanced breast cancer (ABC), with $20 \% 5$-year survival time. ${ }^{3}$ Although $\mathrm{ABC}$ is not curable, ${ }^{4}$ patients can be treated with systemic therapy to control the symptoms of the disease, possibly prolonging survival time and maintaining a better quality of life. Endocrine therapy, representing the standard of care for first-line treatment, ${ }^{5}$ plays a crucial role in $\mathrm{ABC}$ in postmenopausal women 
with hormone receptor-positive $(\mathrm{HR}+)$ disease. ${ }^{6}$ The firstline endocrine therapy consists of tamoxifen, fulvestrant, and third-generation aromatase inhibitors (AIs), such as anastrozole, letrozole, and exemestane., ${ }^{7,8}$

Third-generation AIs, with improved time to progression (TTP) and better tolerability compared with tamoxifen, are considered the standard of care for first-line treatment. ${ }^{9-13}$ Fulvestrant is a selective estrogen receptor (ER) downregulator, which binds, blocks, and increases the degradation of ER proteins, inhibiting estrogen signaling. ${ }^{14}$ The $500 \mathrm{mg}$ dose of fulvestrant was approved based on data from an international, randomized, double-blind, Phase III trial by the name of Fulvestrant and Anastrozole Compared in Hormonal Therapy Naive Advanced Breast Cancer (FALCON). The trial compared fulvestrant $500 \mathrm{mg}$ with anastrozole $1 \mathrm{mg}$ in patients with $\mathrm{HR}+$ locally advanced or metastatic breast cancer who had not received previous endocrine therapy. ${ }^{15}$ In FALCON, progression-free survival (PFS) was significantly longer in the fulvestrant group than in the anastrozole group (HR=0.797, 95\% CI: 0.637-0.999, $P=0.0486)$.

Additionally, cyclin-dependent kinase (CDK)4 and CDK6 inhibitors have shown activity in ER-positive $(\mathrm{ER}+)$ breast cancer in either preclinical or clinical trials. ${ }^{16}$ Palbociclib is the first CDK4/6 approved by the Food and Drug Administration (FDA) for advanced or metastatic breast cancer. ${ }^{17}$ Clinical data favoring palbociclib plus letrozole rather than letrozole alone was suggested in PALOMA-2, a double-blind Phase III study in the initial treatment of postmenopausal women with $\mathrm{ER}+$, human epidermal growth factor receptor 2-negative (HER2-) ABC. ${ }^{18}$ In terms of median PFS, the palbociclib plus letrozole group presented a longer median PFS than letrozole alone ( 24.8 months vs 14.5 months, $\mathrm{HR}=0.58$; 95\% CI: 0.46-0.72; $P<0.000001)$. Beyond that, ribociclib, as a small-molecule inhibitor of CDK4/6, combined with letrozole, could also extend the median PFS versus letrozole alone $\left(\mathrm{HR}=0.56\right.$; 95\% CI: 0.43-0.72). ${ }^{19}$

There are many first-line endocrine therapy regimens at present; however, the optimal sequence for $\mathrm{HR}+\mathrm{ABC}$ is still not established. In a previous network meta-analysis (NMA), the authors examined the comparative effect of different endocrine monotherapies only in PFS/TTP and objective response rate (ORR). ${ }^{20}$ Nonetheless, endocrine combinations are also used to achieve better clinical efficacy. Therefore, this NMA aims to summarize the direct and indirect randomized controlled trials (RCTs) for different first-line endocrine therapies to provide the highest level of evidence for clinicians making decisions in patients with HR+/HER2- ABC.

\section{Materials and methods Literature and search strategy}

We performed this NMA based on the Preferred Reporting Items for Systematic Reviews and Meta-Analysis extension to NMA. ${ }^{21}$

We searched PubMed, EMBASE, and the Cochrane Library for RCTs from inception up to November 21, 2017. The search for titles/abstracts was restricted to English language articles. We used the Medical Subject Headings/Emtree combined with free text words of breast cancer, advanced or metastatic, randomized controlled trial, and all known spellings of tamoxifen, anastrozole, letrozole, exemestane, fulvestrant, palbociclib, and ribociclib. (Detailed information of search strategies in different databases is shown in Figures S1, $\underline{\mathrm{S} 2}$ and $\underline{\mathrm{S} 3}$ ). Additionally, reference lists of eligible published clinical trials and meta-analyses were also tracked manually to identify other relevant studies.

\section{Selection criteria}

Inclusion criteria were drawn based on the framework of population, intervention, comparison, outcome and study design. ${ }^{22}$ The type of participants was $\mathrm{HR}+(\mathrm{ER}+$ and/or progesterone receptor-positive) postmenopausal women in ABC (inoperable locally ABC and metastatic breast cancer), ${ }^{7}$ who had not received previous treatment for the advanced disease or had not received endocrine therapy as an adjuvant treatment within 12 months before study entry. The type of intervention was assessing the effectiveness of one of the following treatments as a monotherapy or in combination as the first-line treatment: tamoxifen, anastrozole, letrozole, exemestane, fulvestrant, palbociclib, and ribociclib. The type of primary outcome was the ORR and PFS/TTP. The type of study was RCTs that evaluated different endocrine therapies as the first-line therapy for treating ABC. We excluded studies where endocrine therapy was used as a neoadjuvant treatment for advanced disease. Two authors (Tingting Zhang and Wenge Zhao) independently assessed the titles/abstracts of studies to identify whether these studies met the inclusion criteria. In the case of existing discrepancies, the two authors reached consensus via discussion.

\section{Data extraction and quality assessments}

Two authors (Tingting Zhang and Fubin Feng) independently extracted the relevant data using a predefined and standardized data extraction EXCEL spreadsheet. We obtained data based on an intention-to-treat analysis. For crossover trials, only the first period of data was extracted. Two authors 
(Tingting Zhang and Fubin Feng) cross-checked all extracted data, and if there were any disagreements between the two authors, a third author (Chuanxin Zang) resolved them. The following data were extracted: first author's name, publication year, journal, study design, sample size, detailed information of intervention (dose, treatment duration, and frequency), characteristics of participants (such as median age, HR status, HER2 status, prior treatment, disease sites, the percentage of measurable disease, Eastern Cooperative Oncology Group performance status, and so on), duration of follow-up, and primary outcomes (PFS/TTP, ORR).

The risk of bias was assessed by using the Cochrane Collaboration's risk of bias assessment tool, ${ }^{23}$ including random sequence generation; allocation concealment; blinding of participants, personnel and outcome assessment; incomplete outcome data; selective reporting; and other bias. When any discrepancies existed between the two authors, a third author provided arbitration.

\section{Statistical methods}

The HR was calculated as the effect size for time-to-event outcomes, and the odds ratio (OR) was calculated for dichotomous outcomes, both with $95 \%$ credible intervals (CrIs). The data of HR or OR with $95 \%$ CI were obtained from the main publications of RCTs. If percentages of ORR were only reported, we needed to convert to decimals to round the numbers.

The data were analyzed using the Markov Chains Monte Carlo method with WinBUGS, Version 1.4.3 (MRC Biostatistics Unit, Cambridge, UK). Three Markov chains were run simultaneously with randomly chosen different initial values. The WinBUGS model ran 150,000 iterations in total, and the iterations of each chain ran 50,000, of which the first 5,000 iterations were a burn-in. When the Deviance Information Criteria (DIC) of the fixed-effect model was smaller, it was used to analyze data. Otherwise, the random-effect model was adopted. ${ }^{24}$ The pooled estimates (HR or OR) were taken to be the median, and $95 \%$ CrIs were presented according to the 2.5 and 97.5 percentiles from the calculated data. We assessed significance according to whether the $\mathrm{CrI}$ included 1 . The surface under the cumulative ranking curves (SUCRA) value was used to rank the treatments for an outcome. If the SUCRA value of interventions is closer to 100 , it indicates that it is always in the first place, and if it is close to 0 , it is always at the end. ${ }^{25}$ When a loop in three arms existed, we adopted a nodesplitting method to evaluate the inconsistency between direct and indirect comparisons. ${ }^{26}$ Individual studies of risk of bias were evaluated by Review Manager, Version 5.3 (The Nordic
Cochrane Centre; The Cochrane Collaboration, Copenhagen, Norway). Further analysis was done by STATA Version 13.0 (Stata Corporation, College Station, TX, USA).

\section{Results Overview of the literature search}

Overall, 3,555 records were identified by the electronic database search, of which 1,189 articles were duplicates. We excluded 2,310 articles that obviously did not meet inclusion criteria after scanning the titles/abstracts. The full text of 56 potentially eligible articles was reviewed, with 41 articles excluded because of the following reasons: non-RCTs $(n=4)$, inappropriate participants $(n=6)$, no data on outcome $(n=11)$, only one study $(n=19)$, and duplicate $(n=1)$, ultimately leaving 15 eligible studies for inclusion. Furthermore, we added another trial to the reference list tracking of clinical trials and meta-analyses. In total, we included 16 articles (14 RCTs) in the NMA. ${ }^{9,11,13,15,17,18,27-36}$ The details of our literature search are shown in Figure 1.

\section{Characteristics of included studies}

Sixteen articles (14 RCTs) published between 2000 and 2017, consisting of a total number of 6,602 participants with $\mathrm{ABC}$ were included in this NMA. The median age of participants across studies ranged from 60.2 to 72.6. Sixteen articles comprising $14 \mathrm{RCTs}$, including 10 different treatment regimens were assessed: anastrozole, letrozole, exemestane, tamoxifen, tamoxifen $40 \mathrm{mg}$, palbociclib+letrozole, ribociclib+letrozole, anastrozole+fulvestrant (loading dose: fulvestrant $500 \mathrm{mg}$ intramuscular on day 1 and $250 \mathrm{mg}$ on days 15 and 29 and after that every fourth week \pm 3 days), fulvestrant $250 \mathrm{mg}$, and fulvestrant $500 \mathrm{mg}$. Among the 14 RCTs, all studies reported a median PFS/TTP and ORR. The percentages of estrogen receptor-positive (ER+) and/or $\mathrm{PgR}+$ were between 43.9 and 100. The median duration of the follow-up ranged from 8.9 months to 49 months. The detailed characteristics of the included trials are shown in Tables 1 and $\underline{\mathrm{S} 1}$.

\section{The assessment of the risk of bias}

The results of the assessment of the risk of bias involving 14 RCTs are presented in Figure 2. Seven RCTs employed double blinding and five RCTs had open-label designs. The remaining papers did not specify whether or not blinding was applied. Most studies did not mention the technique of allocation concealment. Twelve trials were funded by pharmaceutical companies. All studies did not report selectively. 


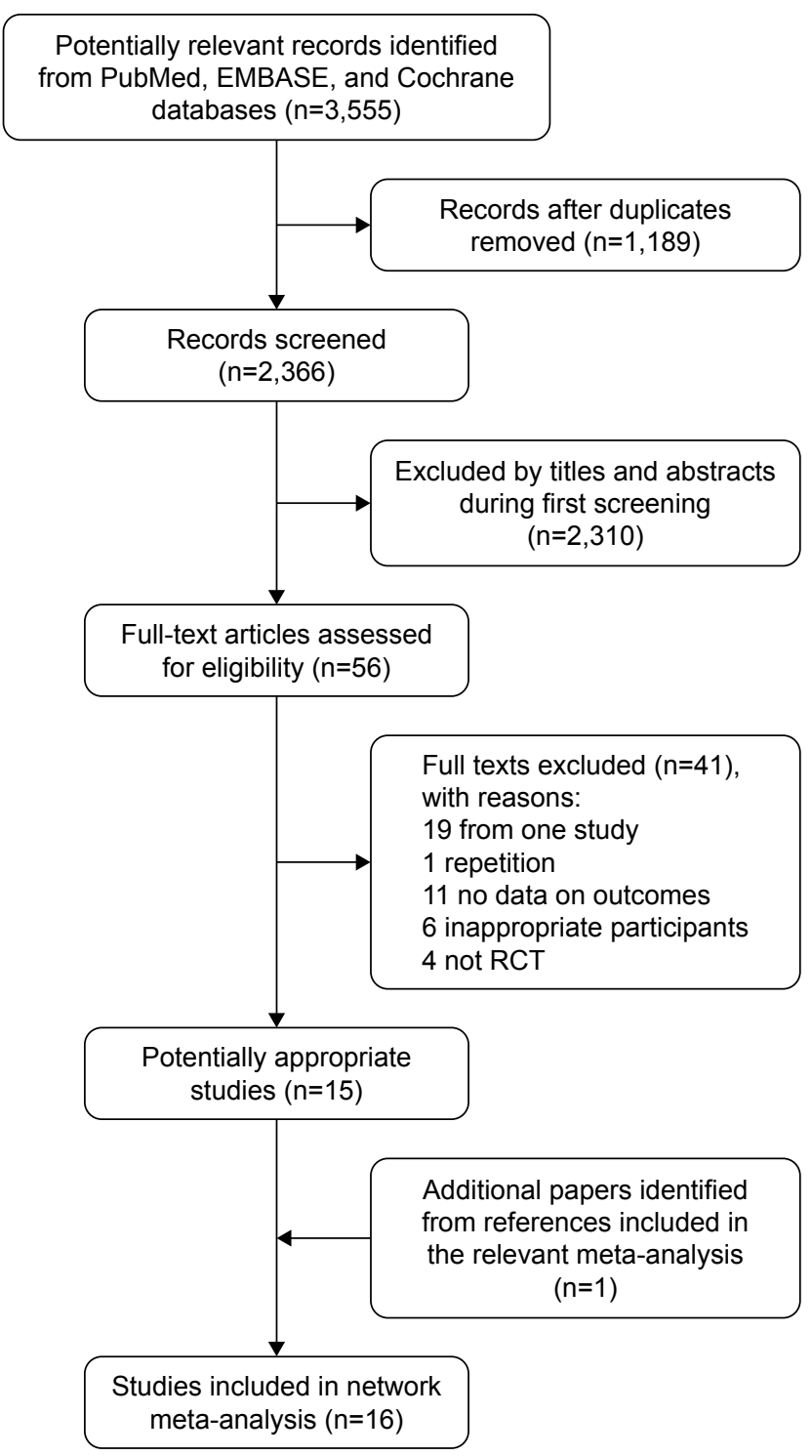

Figure I Flowchart for search results and selection details.

\section{Network meta-analysis}

The networks of all eligible comparisons for primary outcomes of the NMA are shown in Figure 3. The networks for PFS/TTP as a time-to-event outcome and ORR as a dichotomous outcome are the same. Anastrozole was the most frequently involved drug. It was compared with tamoxifen in two trials, anastrozole plus fulvestrant (loading dose) in two trials, exemestane in one trial, fulvestrant $500 \mathrm{mg}$ in one trial, and tamoxifen $40 \mathrm{mg}$ in one trial. As shown in Figure 2, a closed loop existed in anastrozole-exemestanetamoxifen across all comparisons. Based on inconsistency factors (IFs) and 95\% CI, we assessed the inconsistency between direct and indirect evidence. If the value of IF is close to 0 , it indicated that the direct comparison evidence is consistent with the indirect comparison evidence. IFs are 0.196 (95\% CI: 0-0.68) for PFS/TTP and 0.091 (95\% CI: $0-1.05)$ for ORR.

Eight studies reported on TTP and six studies reported on PFS, involving 6,602 patients. The NMA results of PFS/TTP adopted a fixed-effect model (DIC $=-2.249)$ rather than a random-effect model (DIC $=-6.587$ ) according to the size of the DIC value. Palbociclib plus letrozole was superior to anastrozole, letrozole, exemestane, fulvestrant $500 \mathrm{mg}$, tamoxifen, anastrozole plus fulvestrant (loading dose), fulvestrant $250 \mathrm{mg}$, and tamoxifen $40 \mathrm{mg}$ ( $\mathrm{HR}=0.44,95 \% \mathrm{CrI}$ : $0.33-0.58$; HR $=0.56,95 \%$ CrI: $0.45-0.68$; $\mathrm{HR}=0.45,95 \%$ CrI: $0.32-0.61$; HR $=0.58,95 \%$ CrI: $0.42-0.81$; HR $=0.39$, 95\% CrI: $0.30-0.50 ; \mathrm{HR}=0.50,95 \% \mathrm{CrI}: 0.37-0.68 ; \mathrm{HR}=0.33$, 95\% CrI: 0.24-0.45; HR $=0.06$, 95\% CrI: 0.03-0.09; respectively). However, there is no significant advantage compared with ribociclib plus letrozole ( $\mathrm{HR}=1.00,95 \% \mathrm{CrI}$ : 0.72-1.39). Fulvestrant $500 \mathrm{mg}$ was more efficient than other first-line endocrine therapies except for palbociclib plus letrozole and ribociclib plus letrozole ( $\mathrm{HR}=0.75,95 \% \mathrm{CrI}$ : $0.62-0.91$; HR $=0.67,95 \%$ CrI: $0.54-0.83$; $\mathrm{HR}=0.96,95 \%$ CrI: $0.73-1.25$; HR $=0.10,95 \%$ CrI: $0.06-0.16$; HR $=0.57$, 95\% CrI: $0.42-0.76$; HR $=0.77,95 \%$ CrI: $0.58-1.02$; for anastrozole, tamoxifen, letrozole, tamoxifen $40 \mathrm{mg}$, fulvestrant $250 \mathrm{mg}$, and exemestane, respectively). According to the estimated SUCRA values, the four most efficient treatments were palbociclib plus letrozole (SUCRA=94.5\%), ribociclib plus letrozole (SUCRA=94.4\%), fulvestrant $500 \mathrm{mg}$ (SUCRA $=72.1 \%$ ), and letrozole (SUCRA $=68.1 \%$ ), in order. Detailed information is shown in Figure 4A.

In our NMA, all studies reported on ORR. A fixed-effect model (DIC=204.99) was used to analyze the results of ORR. Ribociclib plus letrozole is more effective than the combination of palbociclib and letrozole $(\mathrm{OR}=1.30,95 \% \mathrm{CrI}$ : 0.83-2.02). Even though less clear, benefits were noted with palbociclib plus letrozole and with ribociclib plus letrozole, when compared with other first-line endocrine therapies, with the CrI for OR $>1$ (OR=2.04, 95\% CrI: 1.23-3.36; $\mathrm{OR}=2.39,95 \% \mathrm{CrI}: 1.57-3.67 ; \mathrm{OR}=1.40,95 \% \mathrm{CrI}$ : 1.04-1.89; OR=3.13, 95\% CrI: $1.48-6.63$; OR=2.67, 95\% CrI: $1.54-4.62$; OR $=1.56,95 \%$ CrI: $0.88-2.76$; OR $=1.99$, 95\% CrI: $1.08-3.61$; OR=1.80, 95\% CrI: 0.97-3.31; for anastrozole, tamoxifen, letrozole, tamoxifen $40 \mathrm{mg}$, fulvestrant $250 \mathrm{mg}$, exemestane, fulvestrant $500 \mathrm{mg}$, and anastrozole plus fulvestrant [loading dose], respectively). More NMA results of ORR are summarized in Table 2. As is shown in Figure 4B, the rank of the four most efficient treatments included ribociclib plus letrozole (SUCRA=98.4\%), 


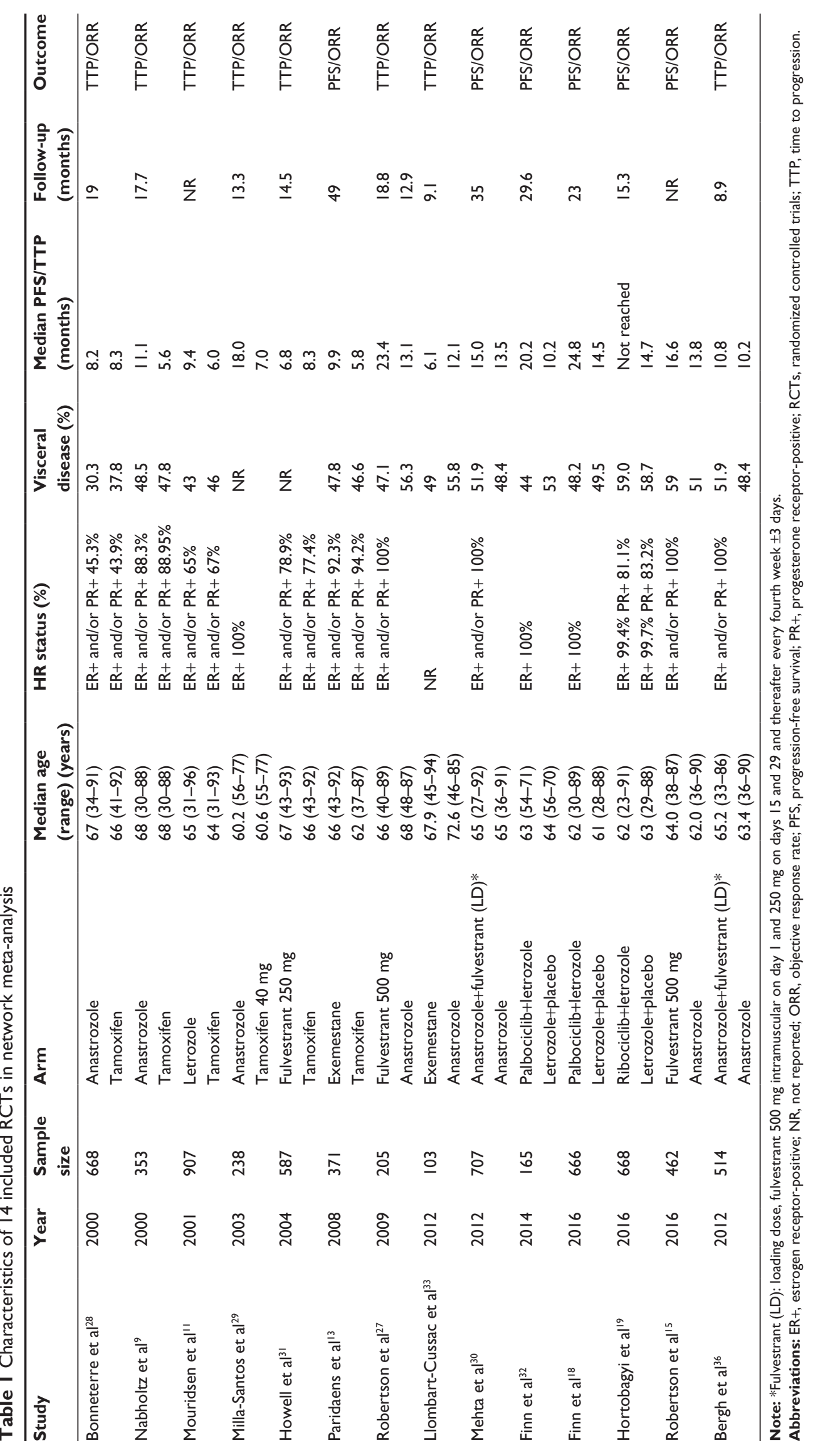




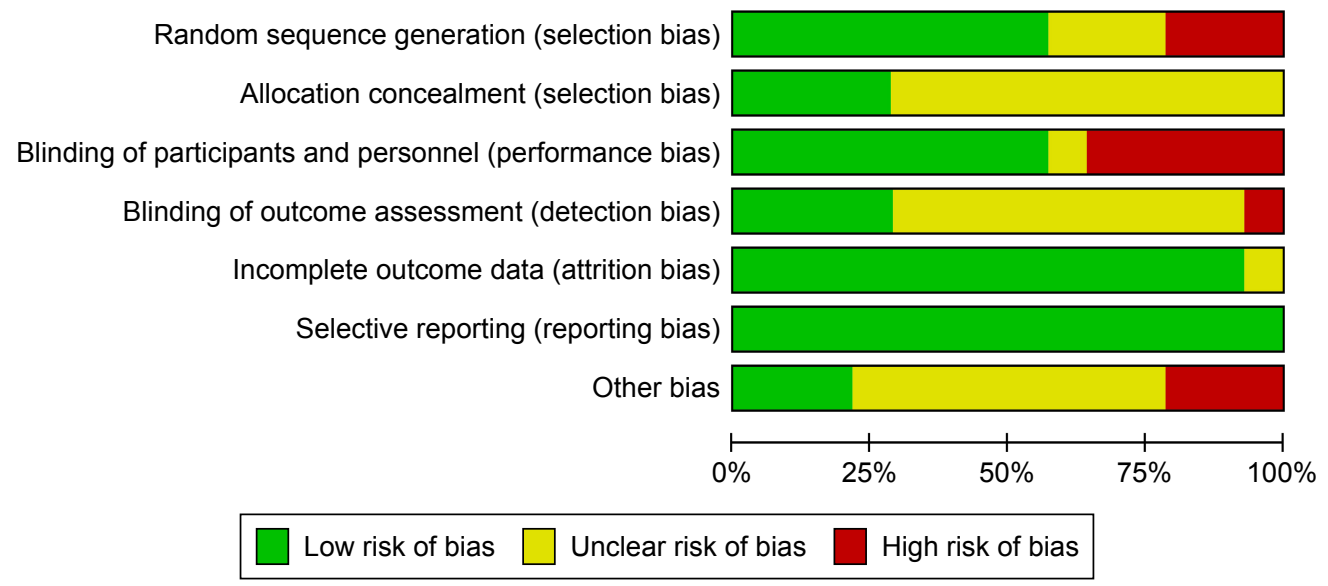

Figure 2 Cochrane risk of bias tool assessment.

palbociclib plus letrozole (SUCRA $=88.9 \%$ ), exemestane (SUCRA $=63.8 \%$ ), and anastrozole plus fulvestrant (loading dose; SUCRA $=52.3 \%$ ) based on the size of the SUCRA value.

\section{Discussion}

Endocrine therapy is the cornerstone treatment for $\mathrm{HR}+\mathrm{ABC}$ patients. AIs are preferred as a first-line therapeutic option for patients with $\mathrm{HR}+\mathrm{ABC}$. With the increasing number of other endocrine therapeutic drugs, such as palbociclib, ribociclib, and fulvestrant, the optimal sequence of first-line treatment for patients with $\mathrm{ABC}$ has not been established. Due to lacking head-to-head RCTs, an indirect comparison of different regimens could be used to assess relative

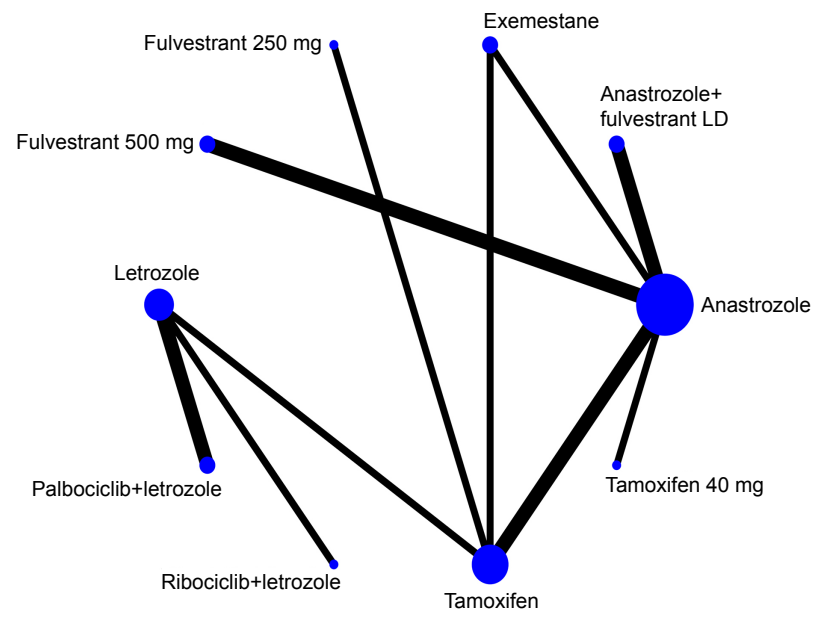

Figure 3 Network of eligible comparisons for network meta-analysis for PFS/TTP. Notes: The width of the lines is proportional to the number of trials comparing every pair of treatments, and the size of every circle is proportional to the number of randomly assigned participants (sample size). The network of eligible comparisons for ORR analysis is also similar.

Abbreviations: LD, loading dose; ORR, objective response rate; PFS, progressionfree survival; TTP, time to progression. efficacy and provide guiding decisions for both physicians and patients. Based on the results of our NMA of different endocrine therapies as a first-line therapy for $\mathrm{ABC}$, statistically significant improvements in PFS/TTP were observed in favor of palbociclib plus letrozole, as well as ribociclib plus letrozole, than other first-line endocrine regimens across all studies. However, regarding the choice of prolonging PFS/TTP, we did not find any significant difference in efficacy between palbociclib plus letrozole and ribociclib plus letrozole. Additionally, the results of the previous NMA are in favor of palbociclib plus letrozole being more efficacious rather than other endocrine therapies and chemotherapy. 37,38 Concerning ORR, ribociclib in combination with letrozole was more effective than either palbociclib plus letrozole or alternative first-line endocrine therapies.

Both palbociclib and ribociclib form part of the selective CDK4/6 inhibitor family. ${ }^{16}$ The CDK4/6-cyclin D-retinoblastoma $(\mathrm{Rb})$ pathway plays an important role in $\mathrm{ER}+$ breast cancer and is the key downstream target of ER signaling. ${ }^{39}$ Therefore, palbociclib and ribociclib inhibit the activity of CDK4/6 kinase and restore its cell cycle control to block the proliferation of tumor cells. ${ }^{38}$ The mechanisms of action are different from fulvestrant, tamoxifen, and AIs. This mechanism may also be the reason that palbociclib or ribociclib could extend the median PFS. Clinical trials of palbociclib plus letrozole in patients with ER+/HER2- ABC showed not only a significantly improved median PFS but also an increased clinical benefit rate. ${ }^{18,32}$ However, perhaps, palbociclib and ribociclib belong to the same class of drugs, so there is no significant difference in prolonging the median PFS. In the previous meta-analysis, the compared results for overall PFS were slightly in favor of ribociclib (HR $=1.16,95 \% \mathrm{CI}$ : $0.81-1.66)$ without statistically significant differences. ${ }^{40}$ 
A
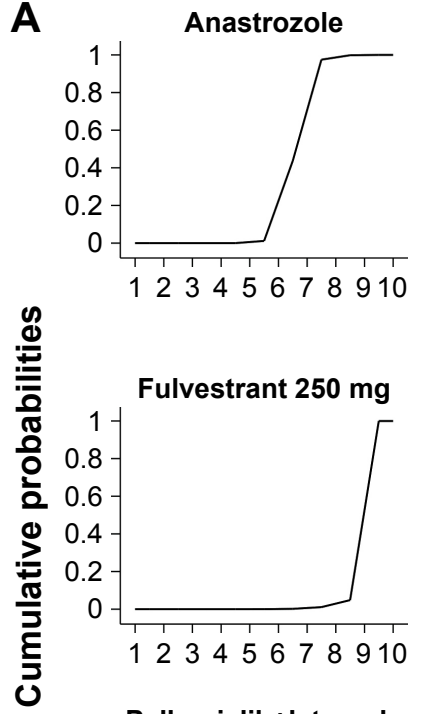

Palbociclib+letrozole

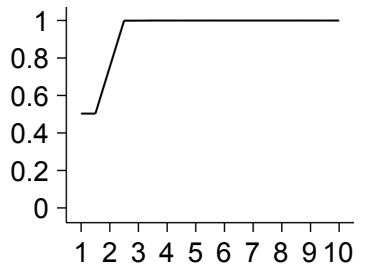

B
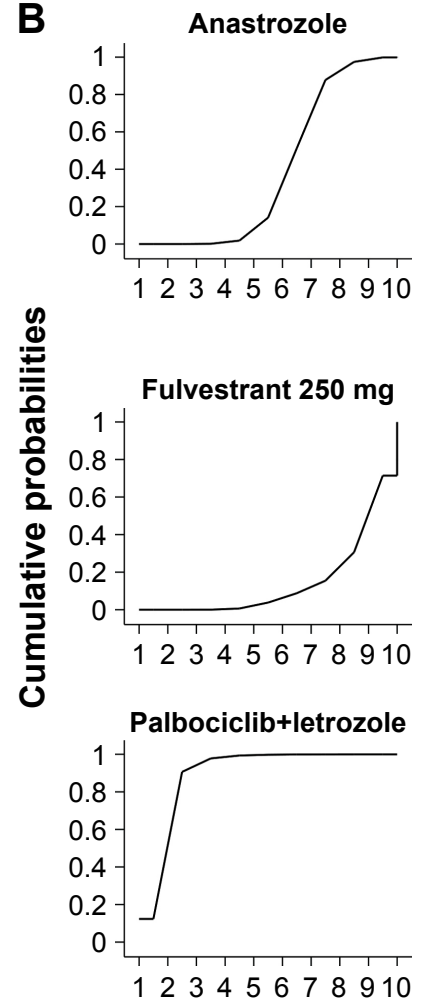

Tamoxifen

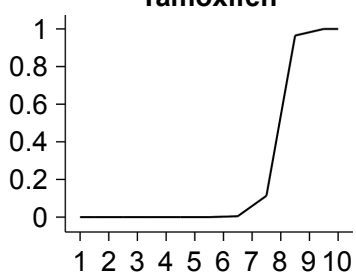

Exemestane
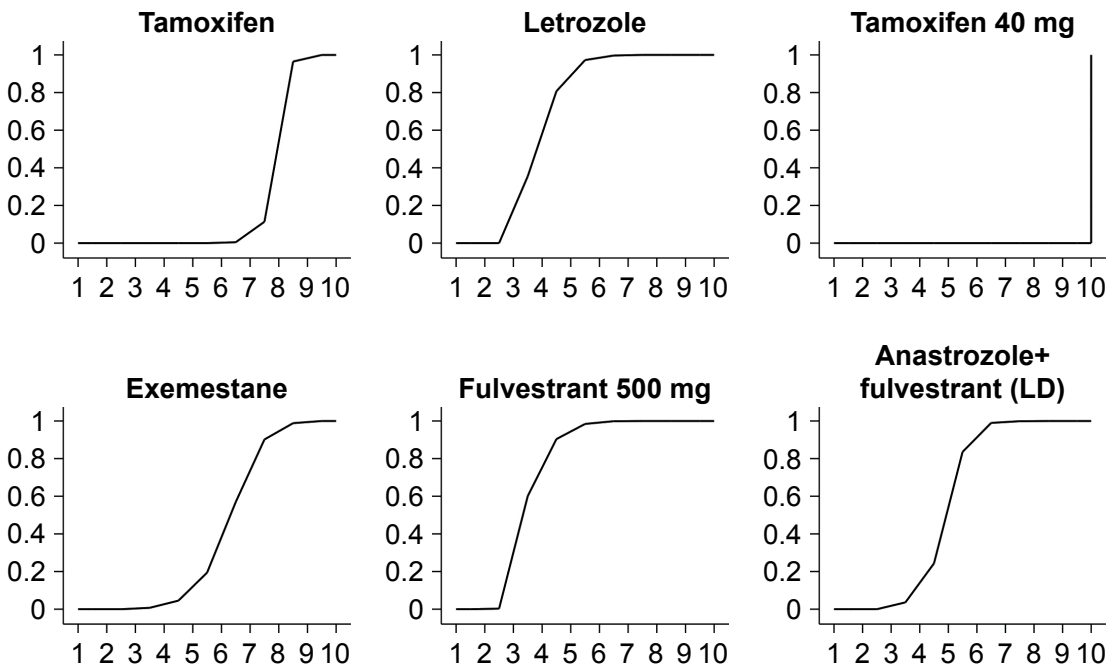

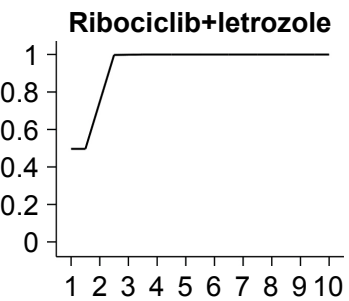

Rank
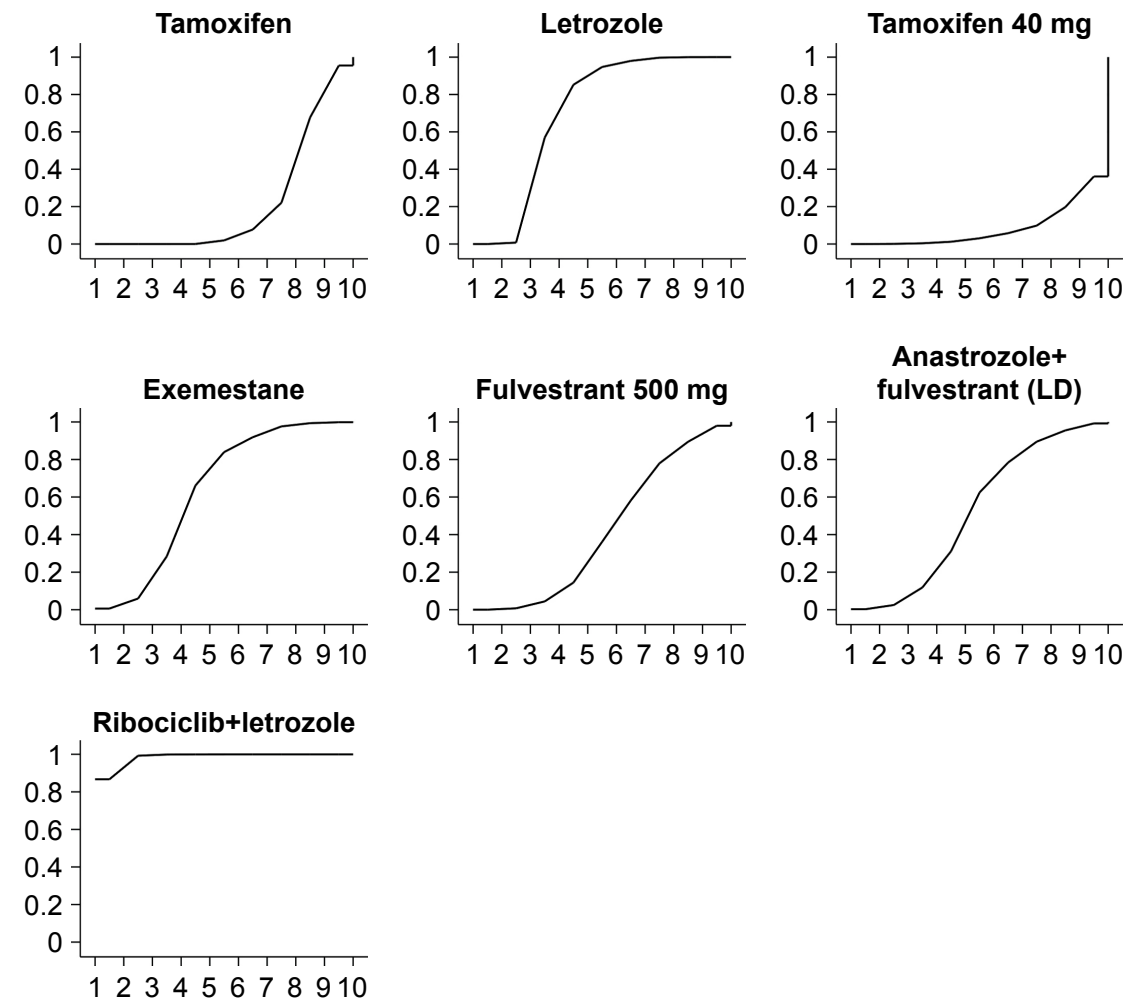

\section{Rank}

Figure 4 The SUCRA for PFS/TTP (A) and ORR (B).

Notes: The larger the SUCRA, the higher the ranking. If the SUCRA value of interventions is closer to 100, it indicates that it is always in first place, and if it is close to 0 , it is always at the end.

Abbreviations: LD, loading dose; ORR, objective response rate; PFS, progression-free survival; SUCRA, surface under the cumulative ranking curves; TTP, time to progression. 


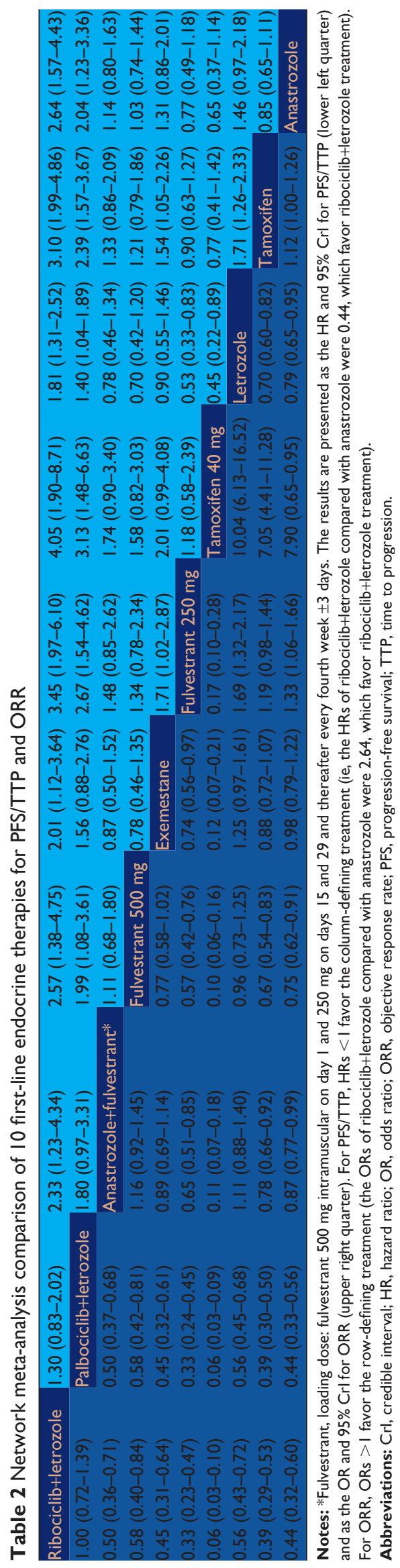

At present, palbociclib or ribociclib in combination with letrozole is approved by the FDA for the first-line treatment of $\mathrm{ABC}$ in postmenopausal patients with HR+/HER2-. ${ }^{41,42}$

Additionally, ribociclib plus letrozole is more efficient than palbociclib plus letrozole regarding ORR. Even though ribociclib and palbociclib are of the same type, there exists a difference between ribociclib and palbociclib since ribociclib could not only inhibit the activity of CDK4/6 pathway but also inhibit cytochrome P3A4 (CYP) and CYP1A2. ${ }^{43}$ This result might lead to the difference in ORR of the two endocrine regimens. Furthermore, concerning adverse events, palbociclib and ribociclib are tolerated better and are associated with a lower risk of treatment-related deaths compared with other conventional cytotoxic chemotherapies and other targeted therapies. ${ }^{44}$ Serious adverse events occurred in $19.6 \%$ of patients in the palbociclib group in PALOMA- 2 and $21.3 \%$ of patients in ribociclib group in MONALEESA-2. ${ }^{18,19}$

\section{Strengths and limitations}

Compared with a previous NMA, our NMA increases the analysis of the application of endocrine combinations in patients with $\mathrm{HR}+\mathrm{ABC} .{ }^{20}$ The results from a previous NMA conducted to evaluate the comparative efficacy of endocrine monotherapy suggested a PFS/TTP benefit for fulvestrant $500 \mathrm{mg} .{ }^{20}$ Our study included not only endocrine monotherapy but also endocrine combinations. Therefore, we have reasons to believe that this NMA is a more comprehensive study for $\mathrm{HR}+\mathrm{ABC}$ and provides a higher level of evidence for both physicians and patients. Certainly, other potential study limitations should be acknowledged. First, our NMA should also be conducted for overall survival; however, these results have been excluded from the current analysis because of immature data in the included clinical trials. Second, due to the lack of unified standards, we did not directly assess specific adverse effects or toxic effects, which limits the confidence with which we can say that palbociclib plus letrozole and ribociclib plus letrozole have the most favorable balance between efficacy and safety. Finally, five of the included studies were open label, which might result in performance bias and measurement bias. Nonetheless, all observed outcomes were objective.

\section{Conclusion}

This NMA showed that palbociclib plus letrozole and ribociclib plus letrozole might be the optimal endocrine therapeutic options for patients with HR+/HER2- ABC. Further investigations of endocrine therapies for $\mathrm{ABC}$ are required to confirm these results and prolong patient survival time. 


\section{Acknowledgments}

The authors would like to acknowledge all the members of the Department of Oncology of Weifang Traditional Chinese Hospital. This study was supported by grants from National Natural Science Foundation of China (Nos. 81473513 and 81673799).

\section{Author contributions}

Changgang Sun was involved in the concept and design of the study. Tingting Zhang drafted the manuscript. All authors participated in acquisition, analysis, and interpretation of the data; revised the manuscript; and read and approved the final version.

\section{Disclosure}

The authors report no conflicts of interest in this work.

\section{References}

1. Jemal A, Center MM, DeSantis C, Ward EM. Global patterns of cancer incidence and mortality rates and trends. Cancer Epidemiol Biomarkers Prev. 2010;19(8):1893-1907.

2. Torre LA, Bray F, Siegel RL, Ferlay J, Lortet-Tieulent J, Jemal A. Global cancer statistics, 2012. CA Cancer J Clin. 2015;65(2):87-108.

3. Gonzalez-Angulo AM, Morales-Vasquez F, Hortobagyi GN. Overview of resistance to systemic therapy in patients with breast cancer. Adv Exp Med Biol. 2007;608:1-22.

4. Cardoso F, Costa A, Senkus E, et al. 3rd ESO-ESMO International Consensus Guidelines for Advanced Breast Cancer (ABC 3). Ann Oncol. 2017;28(1):16-33.

5. Cardoso F, Costa A, Norton L, et al. ESO-ESMO 2nd international consensus guidelines for advanced breast cancer $(\mathrm{ABC} 2)^{\dagger}$. Ann Oncol. 2014;25(10):1871-1888.

6. Mauri D, Pavlidis N, Polyzos NP, Ioannidis JP. Survival with aromatase inhibitors and inactivators versus standard hormonal therapy in advanced breast cancer: meta-analysis. J Natl Cancer Inst. 2006;98(18): 1285-1291.

7. Cardoso F, Costa A, Norton L, et al. ESO-ESMO 2nd international consensus guidelines for advanced breast cancer (ABC2). Breast. 2014;23(5):489-502.

8. Burstein HJ, Temin S, Anderson H, et al. Adjuvant endocrine therapy for women with hormone receptor-positive breast cancer: American society of clinical oncology clinical practice guideline focused update. J Clin Oncol. 2014;32(21):2255-2269.

9. Nabholtz JM, Buzdar A, Pollak M, et al. Anastrozole is superior to tamoxifen as first-line therapy for advanced breast cancer in postmenopausal women: results of a North American multicenter randomized trial. J Clin Oncol. 2000;18(22):3758-3767.

10. Bonneterre J, Buzdar A, Nabholtz JM, et al. Anastrozole is superior to tamoxifen as first-line therapy in hormone receptor positive advanced breast carcinoma. Cancer. 2001;92(9):2247-2258.

11. Mouridsen H, Gershanovich M, Sun Y, et al. Superior efficacy of letrozole versus tamoxifen as first-line therapy for postmenopausal women with advanced breast cancer: results of a phase III study of the International Letrozole Breast Cancer Group. J Clin Oncol. 2001;19(10): 2596-2606.

12. Mouridsen H, Gershanovich M, Sun Y, et al. Phase III study of letrozole versus tamoxifen as first-line therapy of advanced breast cancer in postmenopausal women: analysis of survival and update of efficacy from the International Letrozole Breast Cancer Group. J Clin Oncol. 2003;21(11):2101-2109.
13. Paridaens RJ, Dirix LY, Beex LV, et al. Phase III study comparing exemestane with tamoxifen as first-line hormonal treatment of metastatic breast cancer in postmenopausal women: the European Organisation for Research and Treatment of Cancer Breast Cancer Cooperative Group. J Clin Oncol. 2008;26(30):4883-4890.

14. Robertson JF. Fulvestrant (Faslodex)-how to make a good drug better. Oncologist. 2007;12(7):774-784.

15. Robertson JFR, Bondarenko IM, Trishkina E, et al. Fulvestrant $500 \mathrm{mg}$ versus anastrozole $1 \mathrm{mg}$ for hormone receptor-positive advanced breast cancer (FALCON): an international, randomised, double-blind, phase 3 trial. Lancet. 2016;388(10063):2997-3005.

16. Kwapisz D. Cyclin-dependent kinase 4/6 inhibitors in breast cancer: palbociclib, ribociclib, and abemaciclib. Breast Cancer Res Treat. 2017; 66(1):41-54.

17. New York, NY: Ibrance (palbociclib) prescribing information. Available from: http://www.accessdata.fda.Gov/drugsatfda_docs/ label/2016/207103s002lbl.pdf. Assessed December 30, 2017.

18. Finn RS, Martin M, Rugo HS, et al. Palbociclib and letrozole in advanced breast cancer. N Engl J Med. 2016;375(20):1925-1936.

19. Hortobagyi GN, Stemmer SM, Burris HA, et al. Ribociclib as first-line therapy for HR-positive, advanced breast cancer. $N$ Engl J Med. 2016; 375(18):1738-1748.

20. Zhang J, Huang Y, Wang C, He Y, Zheng S, Wu K. Efficacy and safety of endocrine monotherapy as first-line treatment for hormone-sensitive advanced breast cancer: a network meta-analysis. Medicine. 2017; 96(33): e7846.

21. Hutton B, Salanti G, Caldwell DM, et al. The PRISMA extension statement for reporting of systematic reviews incorporating network meta-analyses of health care interventions: checklist and explanations. Ann Intern Med. 2015;162(11):777-784.

22. Costantino G, Montano N, Casazza G. When should we change our clinical practice based on the results of a clinical study? Searching for evidence: PICOS and PubMed. Intern Emerg Med. 2015;10(4): $525-527$.

23. Higgins JP, Altman DG, Gotzsche PC, et al. The Cochrane Collaboration's tool for assessing risk of bias in randomised trials. BMJ. 2011; 343:d5928.

24. Spiegelhalter DJ, Best NG, Carlin BP, Van Der Linde A. Bayesian measures of model complexity and fit. Ser B Stat Methodol. Epub 2002 Oct 23

25. Salanti G, Ades AE, Ioannidis JP. Graphical methods and numerical summaries for presenting results from multiple-treatment meta-analysis: an overview and tutorial. J Clin Epidemiol. 2011;64(2):163-171.

26. $\mathrm{Lu} \mathrm{G}$, Ades AE. Combination of direct and indirect evidence in mixed treatment comparisons. Stat Med. 2004;23(20):3105-3124.

27. Robertson JF, Llombart-Cussac A, Rolski J, et al. Activity of fulvestrant $500 \mathrm{mg}$ versus anastrozole $1 \mathrm{mg}$ as first-line treatment for advanced breast cancer: results from the FIRST study. J Clin Oncol. 2009; 27(27):4530-4535.

28. Bonneterre J, Thurlimann B, Robertson JFR, et al. Anastrozole versus tamoxifen as first-line therapy for advanced breast cancer in 668 postmenopausal women: results of the tamoxifen or arimidex randomized group efficacy and tolerability study. J Clin Oncol. 2000; 18(22):3748-3757.

29. Milla-Santos A, Milla L, Portella J, et al. Anastrozole versus tamoxifen as first-line therapy in postmenopausal patients with hormone-dependent advanced breast cancer: a prospective, randomized, phase III study. Am J Clin Oncol. 2003;26(3):317-322.

30. Mehta RS, Barlow WE, Albain KS, et al. Combination anastrozole and fulvestrant in metastatic breast cancer. $N$ Engl J Med. 2012;367(5): 435-444.

31. Howell A, Robertson JFR, Abram P, et al. Comparison of fulvestrant versus tamoxifen for the treatment of advanced breast cancer in postmenopausal women previously untreated with endocrine therapy: a multinational, double-blind, randomized trial. J Clin Oncol. 2004; 22(9):1605-1613. 
32. Finn RS, Crown JP, Lang I, et al. The cyclin-dependent kinase $4 / 6$ inhibitor palbociclib in combination with letrozole versus letrozole alone as first-line treatment of oestrogen receptor-positive, HER2-negative, advanced breast cancer (PALOMA-1/TRIO-18): a randomised phase 2 study. Lancet Oncol. 2015;16(1):25-35.

33. Llombart-Cussac A, Ruiz A, Anton A, et al. Exemestane versus anastrozole as front-line endocrine therapy in postmenopausal patients with hormone receptor-positive, advanced breast cancer: final results from the Spanish Breast Cancer Group 2001-03 phase 2 randomized trial. Cancer. 2012;118(1):241-247.

34. Robertson JFR, Lindemann JPO, Llombart-Cussac A, et al. Fulvestrant $500 \mathrm{mg}$ versus anastrozole $1 \mathrm{mg}$ for the first-line treatment of advanced breast cancer: Follow-up analysis from the randomized 'FIRST' study. Breast Cancer Res Treat. 2012;136(2):503-511.

35. Robertson JFR, Llombart-Cussac A, Feltl D, et al. Fulvestrant $500 \mathrm{mg}$ versus anastrozole as first-line treatment for advanced breast cancer: overall survival from the Phase II 'FIRST' study. Cancer Res. 2014;75(9):2997-3005.

36. Bergh J, Jonsson PE, Lidbrink EK, et al. FACT: an open-label randomized phase III study of fulvestrant and anastrozole in combination compared with anastrozole alone as first-line therapy for patients with receptor-positive postmenopausal breast cancer. J Clin Oncol. 2012; 30(16):1919-1925.

37. Chirila C, Mitra D, Colosia A, et al. Comparison of palbociclib in combination with letrozole or fulvestrant with endocrine therapies for advanced/metastatic breast cancer: network meta-analysis. Curr Med Res Opin. 2017;33(8):1457-1466.
38. Wilson FR, Varu A, Mitra D, Cameron C, Iyer S. Systematic review and network meta-analysis comparing palbociclib with chemotherapy agents for the treatment of postmenopausal women with HR-positive and HER2-negative advanced/metastatic breast cancer. Breast Cancer. 2017;166(1):167-177.

39. Goel S, DeCristo MJ, Watt AC, et al. CDK4/6 inhibition triggers anti-tumour immunity. Nature. 2017;548(7668):471-475.

40. Mateo-Carrasco H, Salmen C. Compared efficacy of palbociclib versus ribociclib in advanced breast cancer. J Oncol Pharm Pract. 2017; 23(8):62-63.

41. U.S. Food and Drug Administration. FDA approves Ibrance for postmenopausal women with advanced breast cancer. Available from: https://www.fda.gov/newsevents/newsroom/pressannouncements/ ucm432871.htm. Assessed December 30, 2017.

42. U.S. Food and Drug Administration. Ribociclib (Kisqali). Available from: https:/www.fda.gov/drugs/informationondrugs/approveddrugs/ ucm546438.htm. Assessed December 30, 2017.

43. DiPippo AJ, Patel NK, Barnett CM. Cyclin-dependent kinase inhibitors for the treatment of breast cancer: past, present, and future. Pharmacotherapy. 2016;36(6):652-667.

44. Costa R, Costa RB, Talamantes SM, et al. Meta-analysis of selected toxicity endpoints of CDK4/6 inhibitors: palbociclib and ribociclib. Breast. 2017;35:1-7.
OncoTargets and Therapy

\section{Publish your work in this journal}

OncoTargets and Therapy is an international, peer-reviewed, open access journal focusing on the pathological basis of all cancers, potential targets for therapy and treatment protocols employed to improve the management of cancer patients. The journal also focuses on the impact of management programs and new therapeutic agents and protocols on

\section{Dovepress}

patient perspectives such as quality of life, adherence and satisfaction The manuscript management system is completely online and includes a very quick and fair peer-review system, which is all easy to use. Visit http://www.dovepress.com/testimonials.php to read real quotes from published authors. 